\title{
Structure and Formation of Soot Particles from Tribofilm Decomposition Under Real Engine Conditions
}

Thomas Thersleff ${ }^{\dagger}$, Istvan Zoltan Jenei†, Serhiy Budnyk, Nicole Dörr, and Adam Slabon*

\begin{abstract}
Lubrication of an internal combustion engine is critical for unwanted energy and material losses. Zinc dialkyldithiophosphate (ZDDP) is a commonly used anti-wear additive that forms by in situ decomposition a protecting interface between sliding surfaces. The interface consists of the tribofilm on both surfaces and oil in the contact. Soot particles from a petrol engine and gas engine were analyzed using X-ray photoelectron spectroscopy (XPS) and transmission electron microscopy (TEM) techniques: electron energy loss spectroscopy (EELS) and energy dispersive X-ray spectroscopy (EDS). These techniques revealed that the end-products

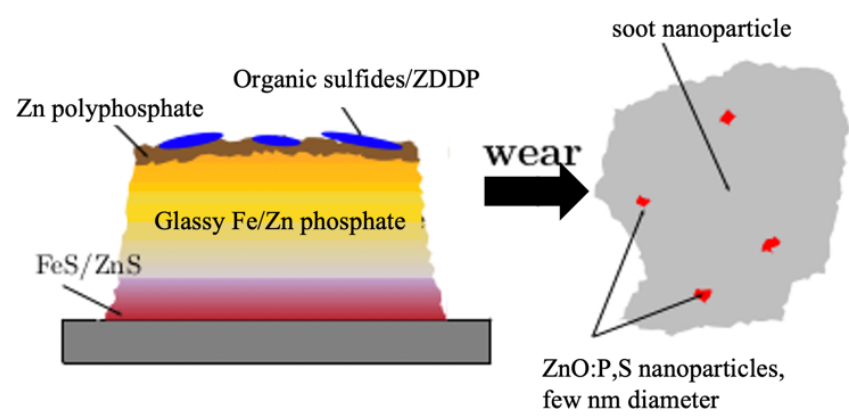
in soot contain 3-5 nm ZnO-based particles with additions of phosphorus and sulfur, originating from the ZDDP anti-wear additive. Our results unravel the tribofilm decomposition under real field conditions and hint toward potentially unidentified hazards with respect to ZDDP-containing lubricants.
\end{abstract}

\section{INTRODUCTION}

It is well established that the lubrication of an internal combustion engine is crucial for reducing unwanted energy and material losses and thus expanding its fuel efficiency and life-time. In order to achieve this, two types of additives, that are mixed into the lubricating engine oil, are critical: friction modifiers and anti-wear additives. ${ }^{1-4}$ The use of the former type of additives have the aim of reducing the friction coefficient between the moving components of an engine, thus reducing the unwanted energy losses. ${ }^{5}$ Examples of such friction modifiers are functionalized polymers, soluble organo-molybdenum additives or dispersed nanoparticles. ${ }^{6}$ The latter type of additives make sure that there is minimal wear inside the engine, so it can function in ideal conditions for an extended period of time, thus to increase its life-time. ${ }^{4}$ Smaller energy loss (i.e. better efficiency) and longer life-time of engines are clearly beneficial. These lead to better fuel economy and, at the same time, less $\mathrm{CO}_{2}$ emissions during running. ${ }^{7}$ According to a study published by Holmberg and Erdemir ${ }^{8}$, the implementation of advanced tribological technologies could reduce $\mathrm{CO}_{2}$ emissions globally as much as $1460 \mathrm{Mt} \mathrm{CO}$. Besides the beneficial effects of friction and wear reduction, it is very important to address the end-products of these lubricants that can be found in the drained engine oils or in the exhaust system.

Zinc dialkyldithiophosphate (ZDDP), as illustrated in Figure 1a, has been commonly used as an anti-wear additive since the middle of the 20th century. 3,9,10 The working mechanism of ZDDP can be described as the following: this additive can form a 50-150 $\mathrm{nm}$ thick tribofilm at relatively low temperatures $\left(50^{\circ} \mathrm{C}\right)^{11}$ on the wear tracks of surfaces sliding against each other. ${ }^{12}$ Initially they form separate patches, which then will evolve into a semi-continuous film, but keeping the pallet-like structure (see Figure 1b). Below the pallets, a sulfur-rich layer is present in form of $\mathrm{Zn} / \mathrm{Fe}$ sulfide. ${ }^{13}$ The most widely accepted anti-wear action of the
ZDDP tribofilm is that it forms a protective barrier between the sliding surfaces, thus preventing a metal-metal contact and consequently wear of the moving components. After the ZDDP tribofilm is formed, the presumed wear originates from the protective film itself. If this mechanism is assumed, the wear debris that is produced has the same chemical composition as the pallet-like tribofilm, i.e. Zn polyphosphate, glassy Fe/Zn phosphate and Fe/Zn sulfide and sulfate. It should be noted that recent reports suggest that the formation of the tribofilm depends on the interface between the ZDDP and substrate. For instance, ZDDP decomposes into binary $\mathrm{ZnO}$ nanoparticles if the substrate is changed from steel to hydrogenated tungsten carbide/amorphous carbon. ${ }^{14}$

A fraction of the worn ZDDP-containing tribofilm fragments (particles) and mechanical wear integrate in a complex matrix of decomposition products as a part of used lubricating oils, and can get into the exhaust system. The phosphate and sulfate components can poison the catalytic converters of the vehicles, reducing the conversion efficiency. ${ }^{15}$ Due to regulations to reduce pollutant content of the exhaust, new methods were developed, e.g. exhaust gas recirculation in case of diesel engines. One side effect of these methods is that they promote the generation of soot nanoparticles (SNPs). These can get into the engine oil and can alter its lubricating properties. ${ }^{16,17}$ Additionally, several studies ${ }^{18-20}$ showed that engine oil additives, such as ZDDP, or their by-products can be adsorbed by these soot nanoparticles, which can also have an effect on the tribological properties of the engine oil. The hardness of these SNPs are in the same order of magnitude as the engine components, ${ }^{21-23}$ which means that an increase of SNPs concentration in the engine oil promotes abrasive wear. If not handled with care, the end-products resulting from the use of ZDDP (parts of the tribofilm and thermal degradation products) might pollute the soil and underground water supplies 
through their $\mathrm{P}$ and $\mathrm{S}$ content. ${ }^{24}$ Although zinc-containing degradation products are characterized by relatively low potential toxicity to the human health in small quantities, higher doses can cause severe intoxication.

a.
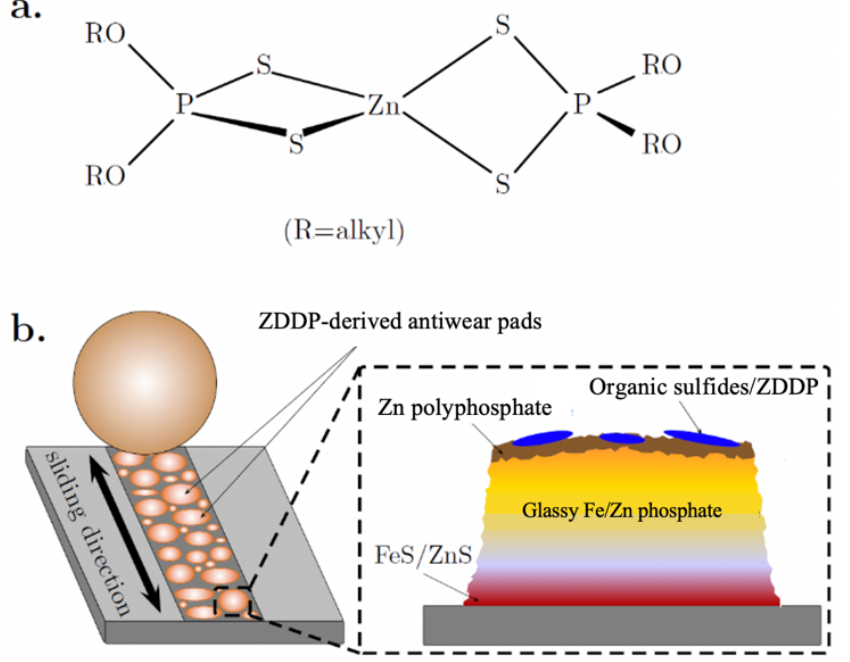

Figure 1. (a) Schematic representation of the zinc dialkylthiophosphate (ZDDP) molecule. (b) Schematic representation of the protectice tribofilm formation from ZDDP additive in a lubrication oil during a ball-on-disk tribotest. Scheme inspired from Spikes ${ }^{3}$ et al.

A study published by Plum et al. ${ }^{25}$ reviews the effects of $\mathrm{Zn}$ exposure to humans: inhaling zinc-containing fumes with particle size $<1 \mu \mathrm{m}$ can cause metal fume fever (MFF). Dermal contact can cause irritations. In rare cases, oral intake of high $\mathrm{Zn}$ dose can even lead to death. Long term exposure to high doses of supplemental zinc interferes with copper uptake of the human body. Due to the increased restrictions on pollutant elements, ${ }^{26}$ such as sulfur and phosphorus, the replacement of ZDDP (which contains considerable amounts of sulfur and phosphorus in form of organic molecules) as an anti-wear additive with other compounds ${ }^{27}$ becomes an issue of high importance. ${ }^{28-31}$ Any newly proposed compound should be able to prevent the production of pollutant end-products, should preserve or improve the antiwear properties while reducing the toxicity and should produce end-products that do not pose an environmental hazard, following the principles of green chemistry. ${ }^{32}$ Previous high-resolution transmission electron microscopy (HRTEM) and scanning electron microscopy (SEM) studies on SNPs have confirmed the overall presence of the elements originating from ZDDP based on SEM energy dispersive X-ray spectroscopy (EDS). ${ }^{33}$ However, the spatial distribution of the ZDDP degradation products within the "bulk" SNPs has remained elusive.

The constant improvement of high performance analytical techniques such as (scanning) transmission electron microscopy (STEM) combined with electron energy loss spectroscopy (EELS) and EDS as well as innovative approach for data processing methods described in our previous work ${ }^{34}$ permitted us in the present work the detection and characterization of the end-products of ZDDP obtained from engine oils. The decomposition of ZDDP with respect to tribofilm formation, i.e. interface between sliding surfaces, has been recently elucidated by means of TEMEDS studies. ${ }^{35}$ However, an understanding of the decomposition process for this formed ZDDP-derived interface and formation of soot particles itself is a matter of debate.

Herein, we present an alternative analytical approach to determine the elemental composition of particles found in a drained engine oil originating from a petrolfueled passenger car, driven under real-life conditions, as well as from a stationary gas engine. The comparative analysis was made using X-ray photoelectron spectroscopy (XPS), EDS and EELS. The latter two techniques were used in a TEM in scanning mode. While the EDS technique provides information about the elemental composition of the sample, EELS, through the Energy-Loss Near-Edge Structure (ELNES) can provide information about the chemical state of the elements. ${ }^{36}$

\section{EXPERIMENTAL}

Passenger car (petrol engine). The sample was selected from a passenger car that was part of a field test. The experimental part and the findings with focus on ZDDP degradation are described in details. ${ }^{5}$ Briefly, the engine was thoroughly rinsed with fresh engine oil to ensure negligible amounts of previously used oil. The field test represented real-life operating conditions in terms of daily commuting, involving a total distance of approximately $110 \mathrm{~km}$ per day, composed of $\approx 20 \%$ city, $\approx 70 \%$ freeway, and $\approx 10 \%$ thoroughfare, respectively. The sampling of the engine oil was performed in predefined intervals so that the entire field test was performed without the need of oil refill. The sample was gained after a total mileage of $19,800 \mathrm{~km}$. Properties and characteristics of the passenger car and the engine oil can be found in Table 1. The sample in this work was gained from the final used oil via centrifugation and filtration. ${ }^{5}$ Therefore, the oil was diluted with $n$-heptane in a mass ratio of approximately $1: 4$ and centrifuged at $40^{\circ} \mathrm{C}, 12,000 \mathrm{rpm}$ for $60 \mathrm{~min}$. After removal of the supernatant solvent, the process was repeated with fresh solvent, in total four times. Finally, the solid residue was dried in an oven. 
Table 1. Characteristics of the passenger car and properties of the engine oil used for the field test.

\begin{tabular}{|c|c|}
\hline Parameter & Description/value \\
\hline \multicolumn{2}{|l|}{ Passenger car } \\
\hline Engine type & $\begin{array}{l}\text { Petrol, } 4 \text { cylinders, turbo- } \\
\text { charged }\end{array}$ \\
\hline Displacement volume (L) & 1.4 \\
\hline Fuel type & $\begin{array}{l}\text { Gasoline RON } 95 \text { see EN } \\
228^{37}\end{array}$ \\
\hline Power at $4200 \mathrm{~min}^{-1}(\mathrm{~kW})$ & 88 \\
\hline Compression ratio (-) & $9.5: 1$ \\
\hline Fuel system & $\begin{array}{l}\text { Seq. multi-port fuel injec- } \\
\text { tors }\end{array}$ \\
\hline Year of manufacture & 2013 \\
\hline $\begin{array}{l}\text { Engine mileage at start of } \\
\text { field test }(\mathrm{km})\end{array}$ & 63391 \\
\hline Main operating mode & Freeway \\
\hline \multicolumn{2}{|l|}{ Engine oil } \\
\hline Engine oil type & SAE $5 W-30$ \\
\hline $\begin{array}{l}\text { Kinem. viscosity at } 40^{\circ} \mathrm{C} \\
\left(\mathrm{mm}^{2} / \mathrm{s}\right)\end{array}$ & 65.8 \\
\hline $\begin{array}{l}\text { Kinem. viscosity at } 100^{\circ} \mathrm{C} \\
\left(\mathrm{mm}^{2} / \mathrm{s}\right)\end{array}$ & 11.4 \\
\hline Viscosity index (-) & 169 \\
\hline Density at $15^{\circ} \mathrm{C}\left(\mathrm{g} / \mathrm{cm}^{2}\right)$ & 0.85 \\
\hline $\begin{array}{l}\text { Total base number } \\
(\mathrm{mg} \mathrm{KOH} / \mathrm{g})\end{array}$ & 6.5 \\
\hline $\begin{array}{l}\text { Neutralization number } \\
(\mathrm{mg} \mathrm{KOH} / \mathrm{g})\end{array}$ & 1.6 \\
\hline Ca content $(\mathrm{mg} / \mathrm{kg})$ & 1910 \\
\hline$P$ content (mg/kg) & 720 \\
\hline S content (mg/kg) & 2350 \\
\hline Zn content $(\mathrm{mg} / \mathrm{kg})$ & 880 \\
\hline
\end{tabular}

Gas engine. The sample from the stationary gas engine was obtained by separation of the solids from oily filter deposits (gas engine continuously operated with SAE 40 oil) according to the following cleaning procedure: $16.5 \mathrm{~g}$ of oily soot was mechanically ground and afterwards washed with 20 $\mathrm{mL}$ toluene. The soot was dispersed with Vortex as well as immersing in an ultrasonic bath for $30 \mathrm{~s}$ and afterwards separated by centrifugation $\left(3500 \mathrm{rpm}, 8 \mathrm{~min}, 25^{\circ} \mathrm{C}\right)$. After decantation of the solvent, the same procedure was repeated two times with toluene, and three times with isopropanol and petroleum ether. The obtained particles were dried over night at $55^{\circ} \mathrm{C}$ and yielded an amount of $11 \mathrm{~g}$ oilfree soot.

Engine oils. The main components of an engine oil additive package comprise ZDDP anti-wear additive, detergents, dispersants and antioxidants. As shown in Table 1, phosphorus, sulfur and zinc can be attributed to ZDDP. Calcium and possibly the remaining sulfur are incorporated in detergents. Calcium occurs as calcium carbonate that provides the base reserve given as total base reserve (TBN). The elements that constitute dispersants and antioxidants are typically not detectable by common elemental analytical methods such as optical emission spectroscopy.
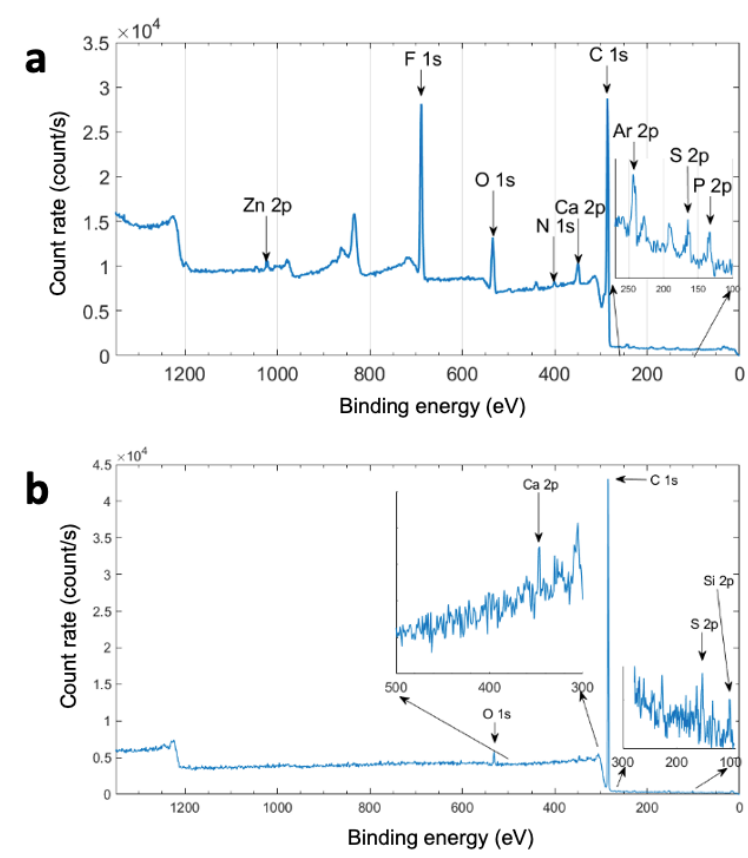

Figure 2. Survey XP spectra of particles originating from (a) petrol engine oil and (b) gas engine oil filter.

X-ray photoelectron spectroscopy (XPS). XPS measurements were performed on a Theta Probe ${ }^{\mathrm{Tm}}$ (Thermo Fisher Scientific). The X-ray source was a monochromated Al $\mathrm{K}_{\alpha}$ source at $1386.6 \mathrm{eV}$. The spectrometer is calibrated to $368.21 \mathrm{eV}$ binding energy (BE) of the $\mathrm{Ag} 3 \mathrm{~d}_{5 / 2}$ line for metallic silver and the linearity is corrected to $\mathrm{BE}$ of metallic $932.62 \mathrm{eV}$ for the $\mathrm{Cu} 2 \mathrm{p}_{3 / 2}$ line and $83.96 \mathrm{eV}$ and $\mathrm{Au} 4 \mathrm{f}_{7 / 2}$. Charge compensation was done using a flood gun for lowenergy electrons and argon ions at $1 \mathrm{eV}$. The binding energy of the corrected spectra are corrected to the main hydrocarbon peak of C $1 \mathrm{~s}$ at $284.6 \mathrm{eV}$ BE. Sputter cleaning and depth profiling was performed by sequentially sputtering with $\mathrm{Ar}^{+}$ ions at $3 \mathrm{kV}, 1 \mu \mathrm{A}, 2 \times 2 \mathrm{~mm}^{2}$. The resolution of the X-ray spot was set to $400 \mu \mathrm{m}$ for all measurements. The survey spectra were recorded with a pass energy of $200 \mathrm{eV} \mathrm{BE}$, the detail spectra with a pass energy of $50 \mathrm{eV}$, respectively. The samples were evacuated in the fast entry lock for several days to maintain the vacuum performance in the analytical chamber. The base pressure in the analytical chamber was $<5 \cdot 10^{-8} \mathrm{~Pa}$. Avantage v5.9917 (Thermo Fisher Scientific) was used to evaluate the XPS spectra. Peaks were fitted using convolution of Lorentzian and Gaussian peak shapes.

Electron microscopy. TEM investigations were carried out using a probe and image Cs corrected Themis $\mathrm{Z}$ operating at $300 \mathrm{kV}$ accelerating voltage. The instrument is equipped with SuperX EDS detector and Gatan Quantum 965 ER dualEELS system. EDS and EELS spectra were acquired simultaneously in STEM mode. The acquired EELS dataset was processed in the following way: first, strong X-ray spikes on the CCD camera were removed by identifying outliers that deviated from the median-subtracted data cube by more than 
6 sigma. The core-loss EELS data cube was subsequently unfolded along the spatial dimensions and decomposed using weighted principal component analysis (PCA) to identify the orthogonal basis best describing the variance of the raw data in a least-squares sense. ${ }^{38,39}$ The rank of the signal subspace was estimated by inspection of the scree plot, and this low-rank approximation was used to generate a spectral model that largely separates the data variance from its noise response. The model was subsequently projected onto the raw data using weighted least squares and then scaled back to the original noise space for chemical analysis. The EELS maps were extracted by employing standard ionization edge extraction routines ${ }^{40}$ on the low-rank PCA approximation of the original data.
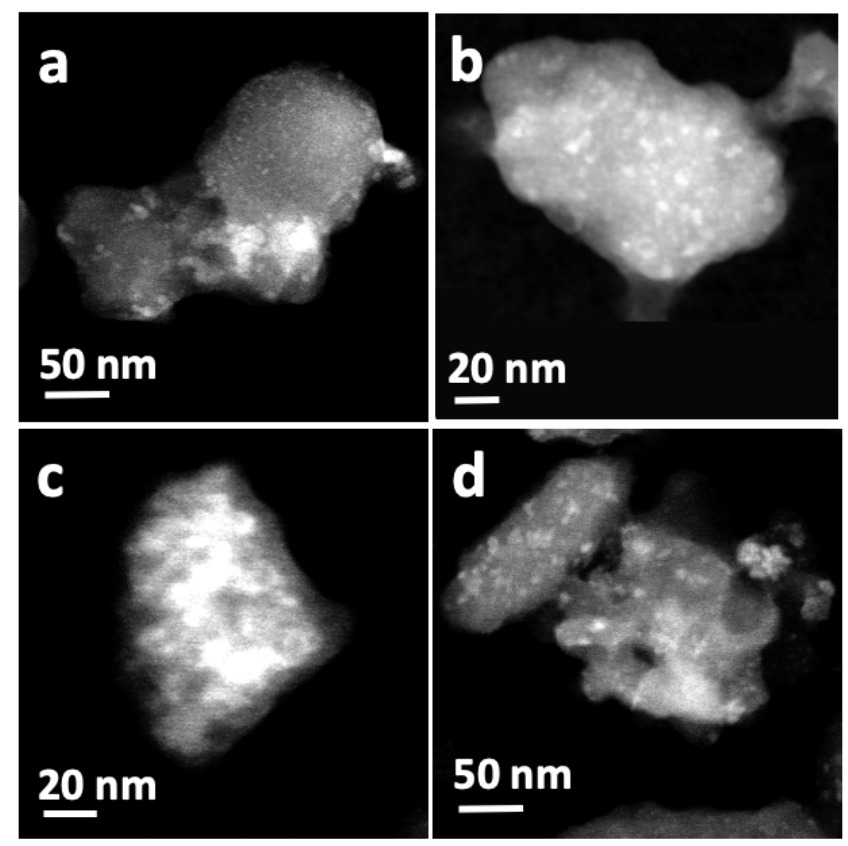

Figure 3. Dark field STEM micrographs of representative particles collected from the petrol engine oil.

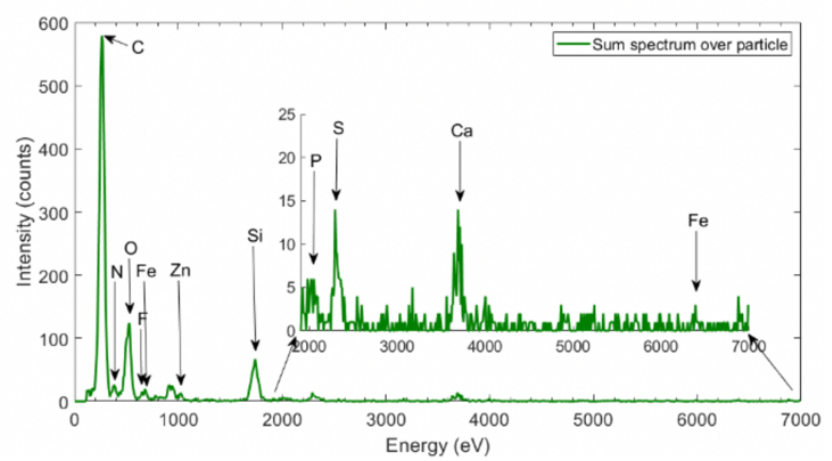

Figure 4. Sum ED spectrum of the particle collected from petrol engine oil (Figure 3a).

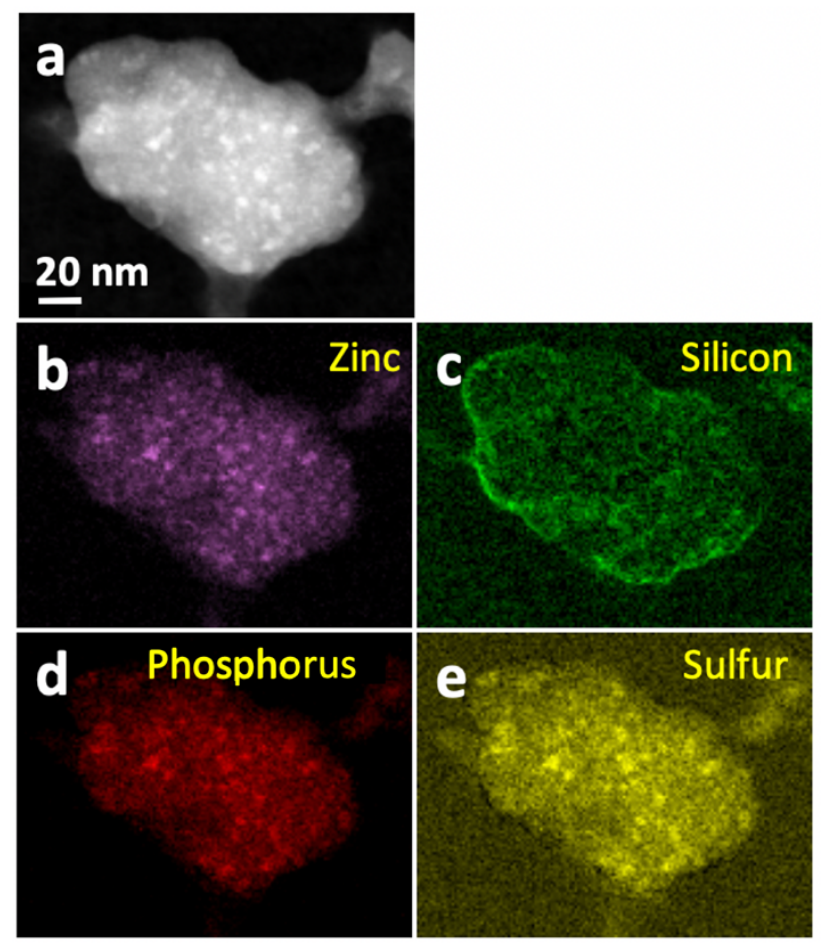

Figure 5. STEM EELS map of the particle collected from the petrol engine oil (Figure $3 b$ ).

\section{RESULTS AND DISCUSSION}

XPS analysis. A low energy-resolution survey spectrum of petrol engine sample revealed the presence of the following elements: carbon, fluorine, oxygen, nitrogen, calcium, phosphorus, sulfur and zinc (Figure 2a). The carbon and partially the oxygen can be explained by the assumption (later proven by TEM) that the sample contains soot particles. Fluorinated additives provide low friction and wear in the engine; ${ }^{41}$ thus, the presence of fluorine can be also explained by the elution or wear originating from fluorinecontaining polymer seals usually applied at elevated temperatures. Nitrogen is known as component of commonly used aminic corrosion inhibitors and dispersants in engine oils preventing sludge formation. Calcium is part of detergents used in engine oil. The zinc, sulfur and phosphorus content can be linked to ZDDP anti-wear additive. 
A depth profile has been made on the sample and the quantitative distribution of the above-mentioned elements are shown in Table 2. Keeping in mind the correspondence between the etching time and the quantity, and thus the thickness of the removed material, it can be assumed that the sample, mostly composed of soot particles, is covered by an oxygen containing layer, i.e. the significant decrease of oxygen content and increase of carbon content observed with increasing of etching time. $\mathrm{Zn}, \mathrm{S}$ and $\mathrm{P}$ are homogeneously distributed in the volume of particles as no significant changes observed with respect to the etching time.

Table 2. Elemental composition of particles collected from the petrol engine oil based on XPS analysis. Relative atomic concentration (at\%).

\begin{tabular}{c|cccccccc}
\hline $\begin{array}{c}\text { Etch } \\
\text { time }\end{array}$ & C 1s & Ca 2p & F 1s & N1s & 0 1s & P 2p & S 2p & Zn 2p \\
(s) & & & & & & & & \\
\hline 0 & 60.5 & 1.0 & 24.5 & 1.4 & 11.2 & 0.7 & 0.5 & 0.3 \\
15 & 69.0 & 1.4 & 18.7 & 1.0 & 8.7 & 0.5 & 0.4 & 0.3 \\
30 & 71.2 & 1.5 & 17.1 & 0.9 & 8.1 & 0.6 & 0.4 & 0.3 \\
45 & 72.2 & 1.5 & 16.1 & 1.1 & 7.7 & 0.6 & 0.4 & 0.3 \\
60 & 72.6 & 1.5 & 15.9 & 1.2 & 7.6 & 0.6 & 0.4 & 0.3 \\
\hline
\end{tabular}

A similar low energy-resolution survey spectrum was also collected on the sample extracted from the gas engine oil (Figure 2b). There are fewer elements detected on this sample: C, O, S, Si and Ca. The abundance of carbon is overwhelming, compared to the sample from the petrol engine. Considering the elemental composition as a function of depth within the sample (seen in Table 3), an oxide layer is at the top of the sample. After $10 \mathrm{~s}$ of etching time the carbon elemental concentration reaches $\approx 99$ at $\%$, while almost all other elemental concentrations decrease to $\approx 0$ at $\%$. This is an indication that the soot originating from the gas engine is much cleaner than the one from the petrol engine due to lower content of additives and much cleaner operation condition with gas as fuel. While XPS is an invaluable tool for surface characterization, it needs to be complemented with other techniques to get spatial information about the distribution of the detected elements.

Table 3. Elemental composition of the particles collected from the gas engine oil filter based on XPS analysis. Relative atomic concentration (at\%).

\begin{tabular}{c|ccccc}
\hline $\begin{array}{c}\text { Etch } \\
\text { time } \\
(\mathrm{s})\end{array}$ & C 1s & 0 1s & S 2p & Si 2p & Ca 2p \\
\hline 0 & 95.0 & 2.5 & 0.5 & 1.5 & 0.5 \\
10 & 98.6 & 0.8 & 0.2 & 0.3 & 0.0 \\
30 & 99.2 & 0.5 & 0.2 & 0.0 & 0.0 \\
50 & 99.7 & 0.0 & 0.3 & 0.0 & 0.0 \\
80 & 99.7 & 0.0 & 0.3 & 0.0 & 0.0 \\
110 & 99.5 & 0.0 & 0.5 & 0.0 & 0.0 \\
140 & 99.5 & 0.3 & 0.2 & 0.0 & 0.0 \\
\hline
\end{tabular}

STEM EELS and EDS analysis. The particles originating from the petrol engine and observed with TEM did not have a specific shape and their size (diameter) were in the range of a few hundred nanometers. STEM micrographs of representative particles can be seen in Figure 3. The contrast in the image indicates that the particle contains small fragments composed of relatively high atomic number elements. The size of these fragments are in the range of 5-10 nm. EDS and EELS spectrum images were collected simultaneously. The sum ED spectrum of a particle collected from the petrol engine (Figure 3a) can be seen in Figure 4. It confirms the presence of the following elements: C, O, Si, P, S, Ca, Fe, F and $\mathrm{Zn}$, and the corresponding quantitative analysis of the overall spectrum is presented in Table 4 . The most abundant element is carbon, which is consistent with the soot nature of the particle, as well as with the XPS results. Besides oxygen, the other elements can be found in relatively small concentrations.

Table 4. Elemental composition of the particle collected from the petrol engine oil (Figure 3a). Relative atomic composition (\%).

\begin{tabular}{c|c|c|c|c|c|c|c|c}
\hline $\mathrm{C}$ & $\mathrm{N}$ & $\mathrm{O}$ & $\mathrm{F}$ & $\mathrm{Si}$ & $\mathrm{P}$ & $\mathrm{S}$ & $\mathrm{Ca}$ & $\mathrm{Zn}$ \\
\hline 84.5 & 0.0 & 11.0 & 1.2 & 2.4 & 0.2 & 0.3 & 0.2 & 0.1 \\
\hline
\end{tabular}

Figure 5 shows the elemental EELS maps of zinc, phosphorus, sulfur and silicon of the particle seen in Figure $3 a$. The EELS spectra were collected in the $800-2800 \mathrm{eV}$ range, which included the following elements: silicon, phosphorus, sulfur and zinc. These elemental maps have a better signalto-noise ratio compared to the EDS maps; thus, they bear more spatial information.

The silicon map indicates that the particle is covered with a silicon-based compound, that can originate from an engine oil additive. ${ }^{42}$ The zinc, phosphorus and sulfur content can be associated with ZDDP as anti-wear additive. The presence of iron (detected in small amounts, as can be seen Figure 4) can be explained by the following hypothesis: iron can originate from the obtained surface tribofilm as a reaction product of the steel surface with active components of the lubricating oil and/or their degradation products. The analyzed fragments of the tribofilm contain a complex composition of iron/zinc phosphate glasses. ${ }^{43}$ The zinc and phosphorus elemental maps shown in Figure 5 suggest the validity of the latter hypothesis. Figure 6 shows the oxygen $\mathrm{K}$ edge extracted from a zinc-rich and from a zinc-free region. Qualitatively, the fine structure of the edge indicates 
the presence of zinc and oxygen. Similar investigation of soot particles obtained by modelling the operation of diesel engine (Patel et al ${ }^{18}$ ) showed presence of zinc sulfate and phosphate in substructure of obtained soot particles.
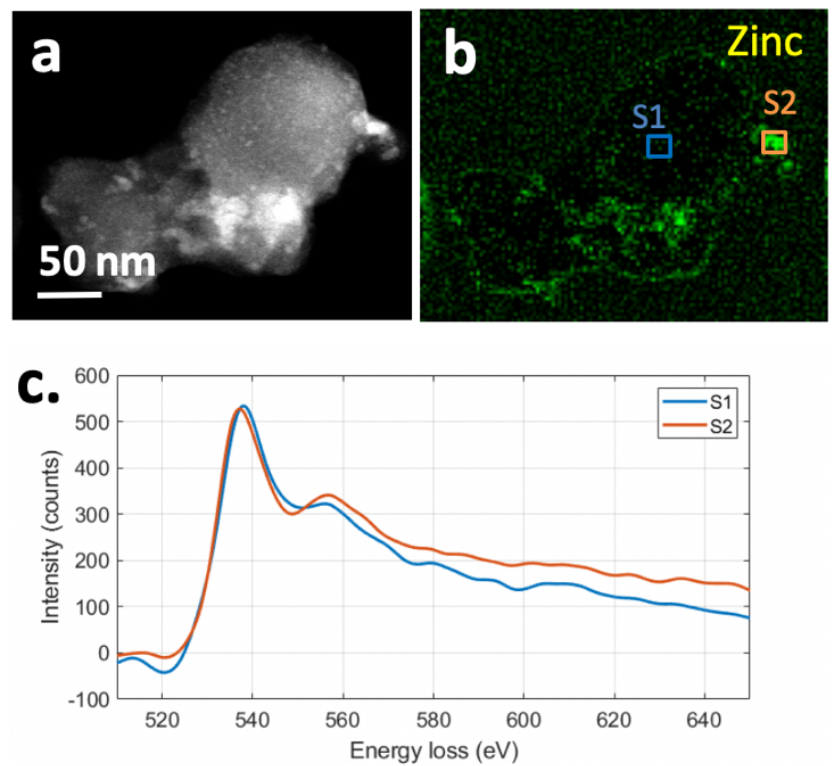

Figure 6. (a) Dark field STEM micrograph of the particle from Figure 3a, (b) Zn elemental map and (c) oxygen K edge extracted from different regions of the particle: S1 from the zinc-free region and S2 from the zinc-rich region.

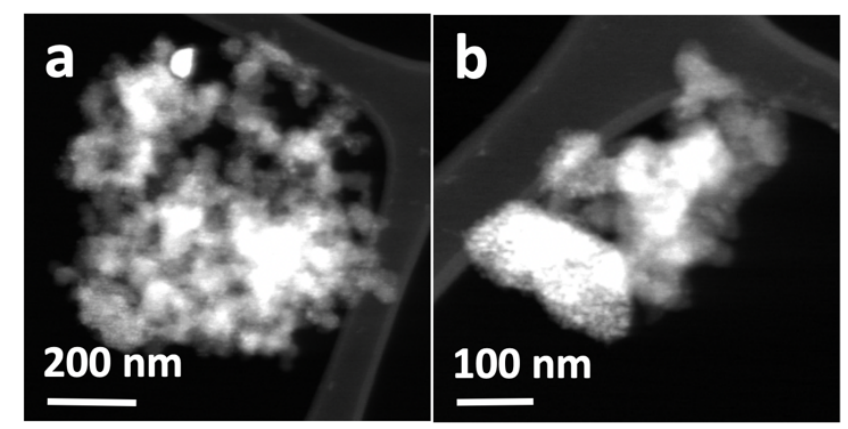

Figure 7. Dark field STEM micrographs of particles collected from the gas engine oil filter.

Figure 7 shows particles found in the soot collected from the gas engine oil filter. Morphology of the particles were found to be similar to particles obtained from the petrol engine oil. The high intensity of the top of the particle (Figure $7 a)$ indicates a presence relatively high atomic number element. The EELS elemental maps (Figure 9) shows that this fragment is rich in $\mathrm{Zn}$ and that its size is in the range of few tens of nanometers. The elemental composition calculated based on the EDS sum spectrum of the particle (see Figure 8 and Error! Reference source not found.) are in good agreement with the results of XPS analysis. Carbon is the most abundant element in the particle, it is distributed homogeneously. The phosphorus, sulfur and zinc elemental maps correlate well with each other (compare with Figure 5), which is a further indication of the presence of zinc sulfate/phosphate.

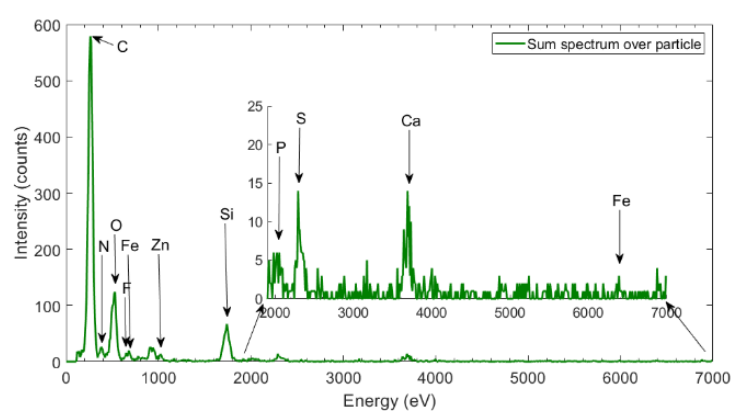

Figure 8. Sum EDS of the particle shown in Figure 7a.

Table 5. Elemental composition at.\% of the particle seen in Figure $7 \mathrm{a}$ based on the EDS sum spectra.

\begin{tabular}{l|l|l|l|l|l|l|l}
\hline $\mathrm{C}$ & $\mathrm{O}$ & $\mathrm{Si}$ & $\mathrm{P}$ & $\mathrm{S}$ & $\mathrm{Ca}$ & $\mathrm{Fe}$ & $\mathrm{Zn}$ \\
\hline 91.0 & 7.6 & 0.1 & 0.2 & 0.5 & 0.5 & 0.0 & 0.1 \\
\hline
\end{tabular}
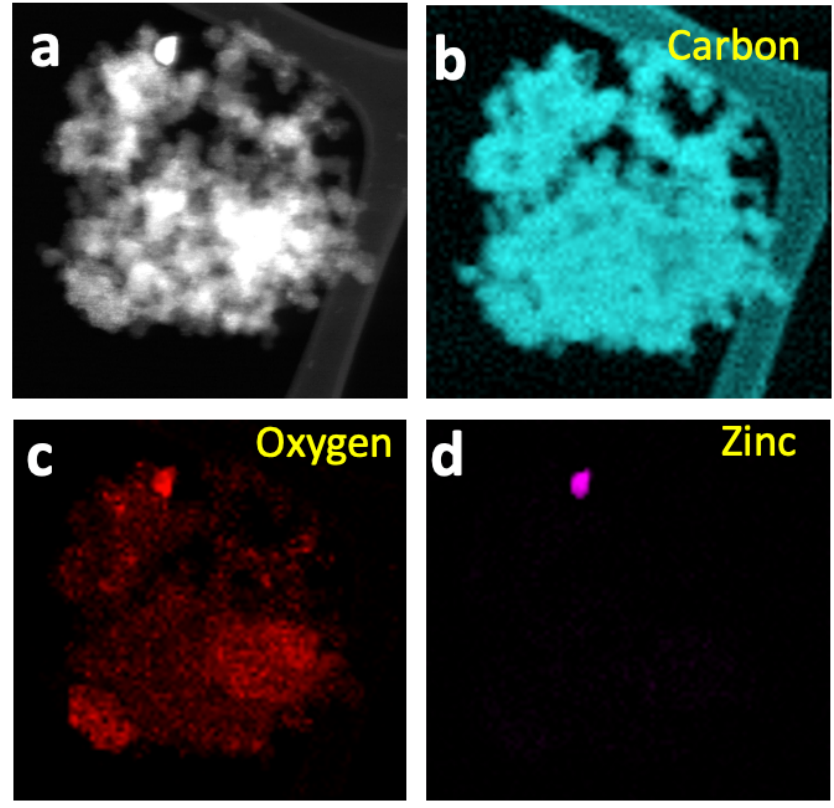

Figure 9. STEM EELS map of the particle shown in Figure 7a.

Figure 10 shows the scheme of a proposed decomposition pathway starting from the tribofilm formed from ZDDP on the surface of the sliding components of the internal combustion engine. Ideally, the tribofilms on both sliding surfaces form a barrier between the sliding surfaces and the only wear will occur from the tribofilm. ${ }^{3}$ Small fragments of the tribofilm (few nm in diameter) thus can enter the combustion chamber and can be incorporated into soot nanoparticles. Agglomerated soot particles, in turn, can be removed from the system by the oil filter system. Particles smaller than a critical size particles can be dispersed in the engine oil by interaction with stabilizing additives.

The above presented analysis shows the presence of zinc in the drained engine oil in form of oxide or mixed phosphate/sulfate glass. While there are methods proposed and developed for removing hazardous elements from used 
engine oils, ${ }^{44}$ the removal of sulfur, phosphorus and (heavy) metals pose challenges. Zinc-containing nanoparticles having diameters in the range of tens of nanometers, pose an increased hazard if released into the environment. From this point of view, it would be of a benefit to replace the ZDDP additive with a "green" lubricant that could provide similar tribological performance to the engine, while it would not produce polluting compounds to the environment.

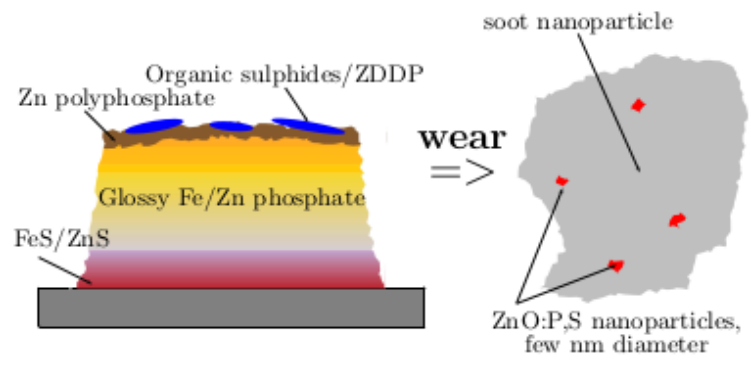

Figure 10. Schematic representation of the suggested metal-ZDDP interface, i.e. tribofilm decomposition mechanism.

\section{CONCLUSION}

The soot composition in real operation conditions of a petrol engine and a gas engine was investigated by the collection of soot from oil and filter during the overall life cycle of motor lubricating oil. The study covered a total mileage of $19,800 \mathrm{~km}$ within 8 months. The degradation of forming tribofilm sheds light on the chemical nature of additive package composition, interaction of base oil with residuals of fuel forming soot particles. The end-products found in the drained lubricating oil from a petrol engine were analyzed using XPS, STEM EDS and STEM EELS. The obtained solid product was compared with the soot originating from a gas turbine engine to evaluate the effect of additives onto chemical composition and morphology. The critical advancement of this work is the discovery of 3-5 $\mathrm{nm} \mathrm{ZnO}$ nanoparticles that contain heterogeneously distributed phosphorus and sulfur within their structure. These ZnO:P,S nanoparticles contain phosphorus and sulfur which originate from the ZDDP anti-wear additive. In case of the petrol engine, elements originating from the oil additives have additional impurities, because $\mathrm{Ca}, \mathrm{F}$ or $\mathrm{N}$ could be detected. Our results unravel the interface decomposition under real field conditions and hint toward potentially unidentified hazards with respect to life-cycle assessment of ZDDP-containing lubricants.

\section{AUTHOR INFORMATION}

\section{Corresponding Author}

Adam Slabon - Department of Materials and Environmental Chemistry, Stockholm University, Svante Arrhenius väg 16 C, 10691 Stockholm, Sweden; ORCID: 0000-0002-4452-1831 Email: adam.slabon@mmk.su.se

\section{Authors}

Thomas Thersleff - Department of Materials and Environmental Chemistry, Stockholm University, Svante Arrhenius väg 16 C, 10691 Stockholm, Sweden

ORCID: 0000-0002-0999-3569

Istvan Zoltan Jenei - Department of Materials and Environmental Chemistry, Stockholm University, Svante Arrhenius väg 16 C, 10691 Stockholm, Sweden

Serhiy Budnyk - AC2T research GmbH, Viktor-Kaplan Str. 2/C, Wiener Neustadt, Austria

Nicole Dörr - AC2T research GmbH, Viktor-Kaplan Str. 2/C, Wiener Neustadt, Austria

ORCID: 0000-0002-8378-4589

\section{Author Contributions}

†These authors contributed equally. All authors have given approval to the final version of the manuscript.

\section{ACKNOWLEDGMENTS}

The authors acknowledge MISTRA (project: SafeChem) for financial support. N.D. acknowledges additional support by the project COMET InTribology (FFG no. 872176, project coordinator: AC2T research $\mathrm{GmbH}$ ).

\section{REFERENCES}

(1) Tang, Z.; Li, S. A Review of Recent Developments of Friction Modifiers for Liquid Lubricants (2007-Present). Curr. Opin. Solid State Mater. Sci. 2014, 18 (3), 119-139. https://doi.org/10.1016/j.cossms.2014.02.002.

Khorramian, B. A.; Iyer, G. R.; Kodali, S.; Natarajan, P.; Tupil, R. Review of Antiwear Additives for Crankcase Oils. Wear 1993, 169 (1), 87-95. https://doi.org/10.1016/0043-1648(93)90394-2.

(3) Spikes, H. The History and Mechanisms of ZDDP. Tribol. Lett. $\quad 2004, \quad 17 \quad$ (3), 469-489. https://doi.org/10.1023/B:TRIL.0000044495.26882.b5.

Li, W.; Kumara, C.; Luo, H.; Meyer, H. M.; He, X.; Ngo, D.; Kim, S. H.; Qu, J. Ultralow Boundary Lubrication Friction by Three-Way Synergistic Interactions among Ionic Liquid, Friction Modifier, and Dispersant. ACS Appl. Mater. Interfaces 2020, 12 (14), 17077-17090. https://doi.org/10.1021/acsami.0c00980.

(5) Dörr, N.; Agocs, A.; Besser, C.; Ristić, A.; Frauscher, M. Engine Oils in the Field: A Comprehensive Chemical Assessment of Engine Oil Degradation in a Passenger Car. Tribol. Lett. 2019, 67 (3), 1-21. https://doi.org/10.1007/s11249-019-1182-7.

(6) Spikes, H. Friction Modifier Additives. Tribol. Lett. 2015, 60 (1), 1-26. https://doi.org/10.1007/s11249-015-0589-z.

Kumara, C.; Leonard, D. N.; Meyer, H. M.; Luo, H.; Armstrong, B. L.; Qu, J. Palladium Nanoparticle-Enabled Ultrathick Tribofilm with Unique Composition. ACS Appl. Mater. Interfaces 2018, 10 (37), 31804-31812. https://doi.org/10.1021/acsami.8b11213. 
Friction 2017, 5 (3), 263-284. https://doi.org/10.1007/s40544-017-0183-5.

Barnes, A. M.; Bartle, K. D.; Thibon, V. R. A. A Review of Zinc Dialkyldithiophosphates (ZDDPS): Characterisation and Role in the Lubricating Oil. Tribol. Int. 2001, 34 (6), 389-395. https://doi.org/10.1016/S0301679X(01)00028-7.

(10) Zhang, J.; Ewen, J. P.; Ueda, M.; Wong, J. S. S.; Spikes, H. A. Mechanochemistry of Zinc Dialkyldithiophosphate on Steel Surfaces under Elastohydrodynamic Lubrication Conditions. ACS Appl. Mater. Interfaces 2020, 12 (5), 6662-6676. https://doi.org/10.1021/acsami.9b20059.

(11) Fujita, H.; Glovnea, R. P.; Spikes, H. A. Study of Zinc Dialkydithiophosphate Antiwear Film Formation and Removal Processes, Part I: Experimental. Tribol. Trans. 2005, $48 \quad$ (4), 558-566. https://doi.org/10.1080/05698190500385211.

(12) Fujita, H.; Spikes, H. The Formation of Zinc Dithiophosphate Antiwear Films. Proc. Inst. Mech. Eng. Part J J. Eng. Tribol. 2004, 218, 265-278. https://doi.org/10.1243/1350650041762677.

(13) Bell, J. C.; Delargy, K. M.; Seeney, A. M. ESI02EDSDF4. Proc. 18th Leeds-Lyon Symp. Tribol. Transient Process. Tribol. 1992, 387.

(14) Soltanahmadi, S.; Charpentier, T.; Nedelcu, I.; Khetan, V.; Morina, A.; Freeman, H. M.; Brown, A. P.; Brydson, R.; Van Eijk, M. C. P.; Neville, A. Surface Fatigue Behavior of a WC/AC:H Thin-Film and the Tribochemical Impact of Zinc Dialkyldithiophosphate. ACS Appl. Mater. Interfaces 2019, 11 (44), 41676-41687. https://doi.org/10.1021/acsami.9b14669.

(15) Ferguson, S.; Johnson, J.; Gonzales, D.; Hobbs, C.; Allen, C.; Williams, S. Analysis of ZDDP Content and Thermal Decomposition in Motor Oils Using NAA and NMR. Phys. Procedia 2015, 66, 439-444. https://doi.org/10.1016/j.phpro.2015.05.055.

(16) Green, D. A.; Lewis, R. The Effects of Soot-Contaminated Engine Oil on Wear and Friction: A Review. Proc. Inst. Mech. Eng. Part D J. Automob.Eng. 2008, 222 (9), 16691689. https://doi.org/10.1243/09544070JAUTO468.

(17) George, S.; Balla, S.; Gautam, M. Effect of Diesel Soot Contaminated Oil on Engine Wear. Wear 2007, 262 (9$10)$, $1113-1122$ https://doi.org/10.1016/j.wear.2006.11.002.

(18) Patel, M.; Azanza Ricardo, C. L.; Scardi, P.; Aswath, P B. Morphology, Structure and Chemistry of Extracted Diesel Soot-Part I: Transmission Electron Microscopy, Raman Spectroscopy, X-Ray Photoelectron Spectroscopy and Synchrotron X-Ray Diffraction Study. Tribol. Int. 2012, 52, 29-39. https://doi.org/10.1016/j.triboint.2012.03.004.

(19) Patel, M.; Aswath, P. B. Morphology, Structure and Chemistry of Extracted Diesel Soot: Part II: X-Ray Absorption near Edge Structure (XANES) Spectroscopy and High Resolution Transmission Electron Microscopy. Tribol. Int. 2012, 52, 17-28. https://doi.org/10.1016/j.triboint.2012.02.022.

Motamen Salehi, F.; Morina, A.; Neville, A. Zinc Dialkyldithiophosphate Additive Adsorption on Carbon Black Particles. Tribol. Lett. 2018, 66 (3), 1-7. https://doi.org/10.1007/s11249-018-1070-6.

(21) Jenei, I. Z.; Dassenoy, F.; Epicier, T.; Khajeh, A.; Martini, A.; Uy, D.; Ghaednia, H.; Gangopadhyay, A. Mechanical Characterization of Diesel Soot Nanoparticles: In Situ Compression in a Transmission Electron Microscope and Simulations. Nanotechnology 2018, 29, 085703.

Jenei, I. Z.; Dassenoy, F.; Epicier, T.; Khajeh, A.; Martini, A.; Uy, D.; Ghaednia, H.; Gangopadhyay, A. Mechanical Response of Gasoline Soot Nanoparticles under Compression: An in Situ TEM Study. Tribol. Int. 2019 , 131, 446-453. https://doi.org/10.1016/j.triboint.2018.11.001.

(23) Jao, T.-C.; Li, S.; Yatasumi, K.; Chen, S. J.; Csontos, A. A.; Howe, J. M. Soot Characterisation and Diesel Engine Wear. Lubr. Sci. 2004, 16 (February 2004), 111-126.

(24) Huang, W.; Dong, J.; Li, J.; Hou, B. The Tribological Performance of S-[2-(Acetamido) Benzothiazol-1-Yl] $\mathrm{N}, \mathrm{N}-\mathrm{Dioctyl}$ Dithiocarbamate as an Additive in Rapeseed Oil. Tribol. Lett. 2004, 17 (2), 199-204. https://doi.org/10.1023/B:TRIL.0000032446.30451.df.

(25) Plum, L. M.; Rink, L.; Hajo, H. The Essential Toxin: Impact of Zinc on Human Health. Int. J. Environ. Res. Public Health 2010, 7 (4), 1342-1365. https://doi.org/10.3390/ijerph7041342.

(26) ACEA EUROPEAN OIL SEQUENCES with EXHAUST AFTERTREATMENT DEVICES; 2016; Vol. 4

Deshpande, P.; Dassenoy, F.; Minfray, C.; Jenei, I. Z.; Le Mogne, T.; Thiebaut, B. Effect of Adding TiO2 Nanoparticles to a Lubricant Containing MoDTC on the Tribological Behavior of Steel/Steel Contacts Under Boundary Lubrication Conditions. Tribol. Lett. 2020, 68 (1). https://doi.org/10.1007/s11249-020-1278-0.

Ratoi, M.; Niste, V. B.; Zekonyte, J. WS2 NanoparticlesPotential Replacement for ZDDP and Friction Modifier Additives. RSC Adv. 2014, 4 (41), 21238-21245. https://doi.org/10.1039/c4ra01795a.

Xu, X.; Hu, J. Q.; Xie, F.; Guo, L.; Ma, J.; Yang, S.Z. The Synergistic Antiwear Performances of Organic Titanium Compounds Containing Sulfur with Borate Ester Additive. J. Spectrosc. 2018, 2018. https://doi.org/10.1155/2018/2576896.

(30) Kohlhauser, B.; Ripoll, M. R.; Riedl, H.; Koller, C. M.; Koutna, N.; Amsüss, A.; Hutter, H.; Ramirez, G.; Gachot, C.; Erdemir, A.; Mayrhofer, P. H. How to Get NoWear? A New Take on the Design of in-Situ Formed High Performing Low-Friction Tribofilms. Mater. Des. 2020, 190 , 108519 . https://doi.org/10.1016/j.matdes.2020.108519.

(31) Sharma, V.; Dörr, N.; Erdemir, A.; Aswath, P. B. Antiwear Properties of Binary Ashless Blend of Phosphonium Ionic Liquids and Borate Esters in Partially Formulated Oil (No Zn). Tribol. Lett. 2019, 67, 1-13. https://doi.org/10.1007/s11249-019-1152-0. 

and Practice; Oxford University Press: New York, 1998.

Vyavhare, K.; Bagi, S.; Patel, M.; Aswath, P. B. Impact of Diesel Engine Oil Additives-Soot Interactions on Physiochemical, Oxidation, and Wear Characteristics of Soot. Energy Fuels 2019, 33 (5), 4515-4530. https://doi.org/10.1021/acs.energyfuels.8b03841.

(34) Ma, Z.; Thersleff, T.; Görne, A. L.; Cordes, N.; Liu, Y.; Jakobi, S.; Rokicinska, A.; Schichtl,Z. G.; Coridan, R. H.; Kustrowski, P.; Schnick, W.; Dronskowski, R.; Slabon, A. Quaternary Core-Shell Oxynitride Nanowire Photoanode Containing a Hole-Extraction Gradient for Photoelectrochemical Water Oxidation. ACS Appl. Mater. Interfaces 2019, $11 \quad$ (21), 19077-19086. https://doi.org/10.1021/acsami.9b02483.

(35) Dorgham, A.; Azam, A.; Morina, A.; Neville, A. On the Transient Decomposition and Reaction Kinetics of Zinc Dialkyldithiophosphate. ACS Appl. Mater. Interfaces 2018, $\quad 10 \quad$ (51), 44803-44814. https://doi.org/10.1021/acsami.8b08293.

(36) Lu, C.; Jothi, P. R.; Thersleff, T.; Budnyak, T. M.; Rokicinska, A.; Yubuta, K.; Dronskowski, R.; Kuśtrowski, P.; Fokwa, B. P. T.; Slabon, A. Nanostructured Core-Shell Metal Borides-Oxides as Highly Efficient Electrocatalysts for Photoelectrochemical Water Oxidation. Nanoscale 2020, 12 (5), 3121-3128. https://doi.org/10.1039/c9nr09818f.

(37) ÖNORM EN 228: Kraftstoffe Für Kraftfahrzeuge Unverbleite Ottokraftstoffe - Anforderungen Und Prüfverfahren (Engl:: Automotive Fuels - Unleaded Petrol - Requirements and Test Methods). ÖNORM EN 228 Kraftstoffe für Kraftfahrzeuge - Unverbleite Ottokraftstoffe - Anforderungen und Prüfverfahren (engl. Automot. fuels - Unleaded Pet. - Requir. test methods), Austrian Stand. Vienna 2017.

(38) Keenan, M. R.; Kotula, P. G. Accounting for Poisson Noise in the Multivariate Analysis of ToF-SIMS Spectrum Images. Surf. Interface Anal. 2004, 36 (3), 203-212. https://doi.org/10.1002/sia.1657.

(39) Spiegelberg, J.; Rusz, J.; Leifer, K.; Thersleff, T. The Usage of Data Compression for the Background Estimation of Electron Energy Loss Spectra. Ultramicroscopy 2017, 181, 117-122. https://doi.org/10.1016/j.ultramic.2017.05.017.

(40) Egerton, R. F. An Introduction to EELS. In Electron Energy-Loss Spectroscopy in the Electron Microscope; 2011; pp 1-28. https://doi.org/10.1007/978-1-4419-95834.

(41) Ebnesajjad, S.; Morgan, R. A. Applications of Fluorinated Additives for Lubricants; 2012. https://doi.org/10.1016/b978-1-4377-3461-4.00006-4.

(42) Zhang, B.; Xu, Y.; Gao, F.; Shi, P.; Xu, B.; Wu, Y. Sliding Friction and Wear Behaviors of Surface-Coated Natural Serpentine Mineral Powders as Lubricant Additive. Appl. Surf. Sci. 2011, 257 (7), 2540-2549. https://doi.org/10.1016/j.apsusc.2010.10.019.

(43) Martin, J. M.; Grossiord, C.; Le Mogne, T.; Bec, S.; Tonck, A. The Two-Layer Structure of Zndtp Tribofilms,
Part I: AES, XPS and XANES Analyses. Tribol.Int. 2001, 34 (8), 523-530. https://doi.org/10.1016/S0301679X(01)00029-9.

(44) Riyanto; Ramadhan, B.; Wiyanti, D. Treatment of Waste Lubricating Oil by Chemical and Adsorption Process Using Butanol and Kaolin. IOP Conf. Ser. Mater. Sci. Eng. 2018, 349 (1), 0-6. https://doi.org/10.1088/1757$899 X / 349 / 1 / 012054$. 